\title{
Avaliação da pressão arterial em pacientes com doença cardiovascular estabelecida: comparando os alvos pressóricos do JNC VIII e do estudo SPRINT
}

\author{
Carolina Ferreira Gomes; Nathalia Perleberg Bachettini; Renata \\ Torres Abib; Aline Longo; Bernardete Weber; Eduardo Gehling \\ Bertoldi
}

\section{Resumo}

Introdução: $O$ controle da pressão arterial contribui com a diminuição do risco cardiovascular (CV), porém determinar o alvo pressórico (AP) adequado para cada paciente continua desafiador. O Eighth Joint National Committee (JNC VIII) propôs, em 2014, metas pressóricas de acordo com comorbidades e idade. Contudo, em 2015, foi divulgado o estudo SPRINT que levantou a necessidade de almejar níveis tensionais mais baixos. O objetivo do atual estudo é verificar a prevalência de pacientes considerados dentro dos AP para o tratamento da HAS de acordo com o SPRINT e o JNC VIII, comparando-os.

Métodos: Foram incluídos 68 pacientes hipertensos, com doença aterosclerótica manifesta e idade acima de 45 anos vinculados ao estudo multicêntrico "Efeito do Programa Alimentar Brasileiro Cardioprotetor na redução de eventos e fatores de risco na prevenção secundária para doença cardiovascular: Um Ensaio Clínico Randomizado", coordenado pelo HCor em parceria com o Ministério da Saúde, a partir do PROADI-SUS. Foram avaliadas as variáveis idade, pressão arterial sistólica (PAS) e diagnóstico de diabetes mellitus (DM). Os dados foram analisados no programa Microsoft Excel, através de classificação etária e diagnóstico de DM. Foram considerados como tendo atingido o AP segundo SPRINT, aqueles pacientes que apresentaram PAS até $120 \mathrm{mmHg}$. Com relação ao estabelecido pelo JNC VIII, a PAS foi considerada dentro do AP quando $\leq$ $140 \mathrm{mmHg}$, em pacientes até 59 anos com e sem DM e em pacientes acima de 60 anos com DM, e até $150 \mathrm{mmHg}$ para pacientes com mais de 60 anos sem DM.

Resultados: A maioria dos pacientes eram homens e 31\% com DM. Quando utilizada as metas do JNC VIII, 63,3\% dos pacientes até 59 anos e com DM atingiram o AP, este percentual elevou-se para 90,4\% entre os sem DM. Entre os pacientes acima de 60 anos, $70,5 \%$ daqueles com DM atingem o AP, prevalência que sobe para 89,4\% entre aqueles sem DM. Quando utilizada o AP do SPRINT, 42,5\% dos pacientes sem DM atingem o alvo, e apenas $25 \%$ no grupo com DM. 
Conclusão: AP mais baixos, como o proposto pelo SPRINT, resulta com que um grande número de pacientes seja considerado fora do alvo, o que leva a um maior uso de medicamentos e, consequentemente, a uma maior exposição a paraefeitos e a uma elevação de custo. Contudo, tal proposta pode representar em uma redução do risco CV. 